\title{
Late termination of pregnancy by intracardiac potassium chloride injection: 5 years' experience at a tertiary referral centre
}

\author{
L Govender, J Moodley \\ Department of Obstetrics and Gynaecology, Lower Umfolozi District War Memorial Hospital, Empangeni, and Nelson R Mandela School of \\ Medicine, University of KwaZulu-Natal, Durban \\ L Govender, BSc, MB ChB, FCOG, MMed (O\&G)
}

Women's Health and Research Group, Department of Obstetrics and Gynaecology, Nelson R Mandela School of Medicine, University of KwaZuluNatal, Durban

J Moodley, MB ChB, FRCOG, FCOG, MD

Corresponding author: L Govender (govenderl18@ukzn.ac.za)

\begin{abstract}
Objectives. To report our experience with intracardiac potassium chloride $(\mathrm{KCl})$ injection as a method of feticide for severe congenital abnormalities beyond 24 weeks' gestation.

Method. A retrospective chart review. Patient demographics and types of fetal anomalies were analysed according to the groups that accepted or declined late termination of pregnancy (LTOP, $\geq 24$ weeks) for severe congenital abnormalities.

Results. Of 3896 women referred to the Fetal Medicine Unit at Inkosi Albert Luthuli Central Hospital, KwaZulu-Natal, 2209 (56.7\%) were at $\geq 24$ weeks' gestation at their first visit. LTOP for severe congenital abnormalities was offered to 253 (11.5\%), of whom 191 (75.5\%) accepted. Differences in maternal age, parity, race and religion were not statistically significant. The type of fetal abnormalities and gestational age at diagnosis influenced the decision-making process in $>80 \%$ of the women. The most frequent indications for LTOP were brain and spinal abnormalities (53.0\%), and aneuploidy (20.6\%). Feticide by ultrasound-guided intracardiac $\mathrm{KCl}$ injection was performed in 138/191 cases (72.2\%); 53 women who accepted LTOP did not undergo feticide for a variety of reasons. The mean interval between diagnosis and performance of feticide was 10 days (range $0-42$ days). Fetal asystole was achieved in all cases within 2 minutes by a single-needle injection of intracardiac $\mathrm{KCl}$; the mean duration of the procedure was 12 minutes (range 6 - 25 minutes). There were no maternal complications, and stillbirths occurred in all cases.

Conclusion. Feticide by ultrasound-guided intracardiac $\mathrm{KCl}$ injection was an acceptable, safe and effective method for LTOP. Further studies are needed to determine the minimum dosage of $\mathrm{KCl}$ required to achieve the desired effect.

S Afr Med J 2013;103(1):47-51. DOI:10.7196/SAMJ.6006
\end{abstract}

The South African Choice on Termination of Pregnancy Act, No. 92 of 1996, allows termination of pregnancy (TOP) for severe fetal abnormalities at any gestational age (GA). ${ }^{1}$ Despite the provision of free antenatal care to all pregnant women, the majority of those attending public health institutions 'book' for antenatal care in the late second or third trimester of pregnancy and miss the opportunity for early prenatal diagnosis of major lethal congenital abnormalities and early intervention if required. Limited resources and expertise at public health institutions compound the problem of diagnosing fetal abnormalities in early pregnancy. Consequently, many abnormalities are detected only after the fetus is viable, i.e. at $\geq 24$ weeks' gestation.

Congenital abnormalities likely to result in the birth of a severely mentally or physically handicapped child necessitate discussing outcomes and possible grounds for TOP. Decisions regarding late termination of pregnancy (LTOP) should not involve just pregnant women, their families and their clinician. Ideally there should be a consensus agreement by a multidisciplinary team that governs the decision-making process for approval of an LTOP. The multidisciplinary team must carefully consider the legal and ethical implications of the clinical decision that an anomaly is sufficiently severe for LTOP, ${ }^{2}$ which should take into account social, cultural, religious and psychological viewpoints. Women should also be counselled about the clinical management of pregnancies complicated by severe fetal anomalies. When there is certainty about the diagnosis and the high probability of either death or survival with severe and irreversible handicap as a result of the anomaly diagnosed, the pregnant woman should be offered the alternatives of aggressive versus non-aggressive obstetric management. ${ }^{3}$ It is also ethically permissible to offer feticide followed by TOP after viability in such cases. Ideally, all women should be informed about the need for autopsy and other appropriate investigations following feticide or perinatal death for a severe fetal anomaly, which allows accurate counselling regarding future pregnancies. ${ }^{4}$

Deciding to terminate a viable pregnancy (where there is a chance of ex utero survival) places the women, her family and health professionals (including midwives) in a difficult situation. The chance of a live birth following a medical termination increases with advancing gestation. Crucial neonatal management decisions must be made if these fetuses are born alive. Neonatologists are duty-bound to provide basic palliative care for as long as the infant survives.

Irrespective of the reasons for late termination, ${ }^{5}$ feticide is therefore performed to ensure that a viable fetus is stillborn. Several methods of feticide have been described, ${ }^{6-11}$ including intracardiac or intraumbilical potassium chloride or lignocaine injection to induce cardiac asystole. For women who choose to terminate pregnancy with an abnormal fetus at an advanced gestation, the method used must be safe and effective. We report on our experiences of feticide with intracardiac potassium chloride $(\mathrm{KCl})$ injection for LTOP among 
women who chose this option and the indications for and acceptance of LTOP in this group.

\section{Methods}

Charts of women offered LTOP for severe congenital abnormalities during August 2003 - December 2008 at the Fetal Medicine Unit, Inkosi Albert Luthuli Central Hospital (IALCH), KwaZuluNatal (KZN), were retrospectively reviewed. IALCH is a tertiary/ quaternary referral hospital and the only public health institution that performs LTOP by feticide in this province. Institutional ethical and hospital permission to perform this audit was obtained. The diagnosis of a severe fetal malformation was based on level III ultrasound findings, with or without adjunctive tests such as fetal magnetic resonance imaging and karyotyping. Obstetric ultrasound examinations were performed using the Siemens Acuson S2000 machine, with conventional 2D scan, colour and spectral pulsed-wave Doppler. A fetal medicine specialist (LG) performed all ultrasound scans with assistance from two experienced sonographers.

A severe fetal abnormality was diagnosed by consensus opinion of a multidisciplinary team including fetal medicine specialist, obstetrician, midwife, geneticist/paediatrician, bioethicist, psychologist, social worker, genetic counsellor, and other experts depending on the nature of the case, e.g. cardiologist, paediatric surgeon or neurosurgeon. Counselling before TOP was provided to all the women by two or more trained healthcare workers on two or more occasions. Written informed consent was obtained from those who agreed to LTOP by feticide.

The inclusion criteria included singletons and dichorionic twins with severe fetal abnormalities at $>24$ weeks' gestation. The patient demographics and types of fetal anomalies were analysed according to the groups that accepted or declined LTOP. Reasons for declining LTOP were documented. In the LTOP feticide group, the following parameters were evaluated: GA when the procedure was performed, duration of the procedure, amount of $\mathrm{KCl}$ used to achieve permanent asystole, number of needle insertions to reach target, procedure-related complications, procedure-to-delivery interval, induction versus spontaneous labour, and any labour complications. Information was captured using a clinical software package.

\section{Procedure of feticide}

The procedure was done on an outpatient basis by a fetal medicine specialist. Other members of the team included a doctor (specialist obstetrician or registrar in training) to aspirate heart blood, administer the $\mathrm{KCl}$ and assist with concomitant procedures if indicated, e.g. amniodrainage, cephalocentesis; a midwife to attend to the patient and keep her comfortable during the procedure; a scrub sister to assist with the procedure, including collecting and labelling blood specimens; and a sonographer/assistant to 'drive' the ultrasound machine as required during the procedure.

Owing to rotation of clinical staff and to minimise any chance of errors, the assistant staff were regularly orientated on the invasive procedure and their roles were explained before commencing. The wishes of healthcare workers with personal objections to the performance of feticide were respected. The requirements for the procedure, including needles, heparinised syringes and drugs, were set up by the scrub nursing sister, who is a dedicated staff member of the unit. The expiry date and labelling of all drugs used for the procedure were counter-checked by the fetal medicine specialist before commencing the procedure. A fully equipped resuscitation trolley, which was checked regularly, was available at the clinic. The mother's Rh status was checked before the procedure, and if she was $\mathrm{Rh}$ negative the fetal heart blood $\mathrm{Rh}$ was checked and the patient managed accordingly.

At least half an hour before the feticide procedure, all the patients were given pethidine $50 \mathrm{mg}$ intravenously for sedation and an antiemetic if necessary. After excluding allergies, a second-generation cephalosporin (mefoxin) $2 \mathrm{~g}$ was given intravenously as a prophylactic antibiotic. The fetus was scanned to obtain a four-chamber view of the fetal heart, and the fetal heart rate and procedure commencement time were recorded. The procedure was performed in the scan room under sterile conditions, using the free-hand technique and under continuous ultrasound guidance. Lignocaine $2 \%$ was used to infiltrate the maternal abdominal skin at the site of entry into fetal heart. A 20-gauge $15 \mathrm{~cm}$ spinal needle was traversed through the predetermined tract, targeting the left ventricle or alternatively the most accessible chamber of the fetal heart. On confirming placement of the needle in the fetal heart by direct sonographic visualisation of the needle tip, the assistant removed the stylet. To reduce the risk of feto-maternal contamination through the needle tract during insertion, the initial $1 \mathrm{ml}$ of aspirated heart blood was discarded. A specimen of heart blood was withdrawn for a full blood count, a Kleihauer test, karyotyping and other investigations as indicated, e.g. viral screen (TORCH and parvovirus) and other genetic studies. Thereafter, the assistant doctor injected $2 \mathrm{ml}$ of $15 \%$ $\mathrm{KCl}$ into the fetal heart under direct vision. A further $2 \mathrm{ml}$ was injected every 30 seconds until permanent fetal asystole was achieved, and the time was recorded. The fetal heart was observed on scan for a further 5 minutes of asystole with the needle in situ. To minimise any risk of maternal contamination with $\mathrm{KCl}$, the needle was flushed with $5 \mathrm{ml}$ sterile water or saline before removal.

All patients were observed and their vital signs assessed in the clinic for 1 hour after the procedure. To confirm permanent fetal asystole, a rescan was performed at least 30 minutes after the procedure or just before the patient left the clinic. All 'low-risk' women were referred back to their base hospital for standard obstetric management and delivery including assessment by a social worker and a psychologist, as indicated. Provided there were no contraindications to vaginal delivery, the women were given the choice to have labour induced or to await spontaneous onset of labour. The obstetric management was the same as that of a woman presenting with a spontaneous intra-uterine death after 24 weeks' gestation. This included weekly monitoring of maternal blood coagulation profiles in those managed expectantly to ensure that they did not develop a coagulopathy. Labour was induced if women did not go into spontaneous labour within 3 weeks.

All women were given a 6-week follow-up appointment with the genetic counsellor for on-going counselling and review of the fetal blood results, and to record the perinatal outcome of those who declined LTOP by feticide.

\section{Statistical analysis}

The numerical analysis was performed using the Statistical Package for Social Sciences (SPSS) for Windows version 16.0 (SPSS Inc., Chicago, Ill., USA) to explain the concepts that underpin the analyses. The data were analysed as tables and figures. The groups that accepted and declined TOP were compared with reference to their demographics, types of fetal anomalies and delivery outcome. Frequency tables and cross-tabulations reporting counts and percentages were used to describe the distribution of the data. Pearson's chi-square test was used to compare demographics and diagnoses between groups. Analysis of variance testing with Bonferroni post-hoc tests was used to compare mean dose of $\mathrm{KCl}$ between the GA categories. A $p$-value of $<0.05$ was considered to be statistically significant. 


\section{RESEARCH}

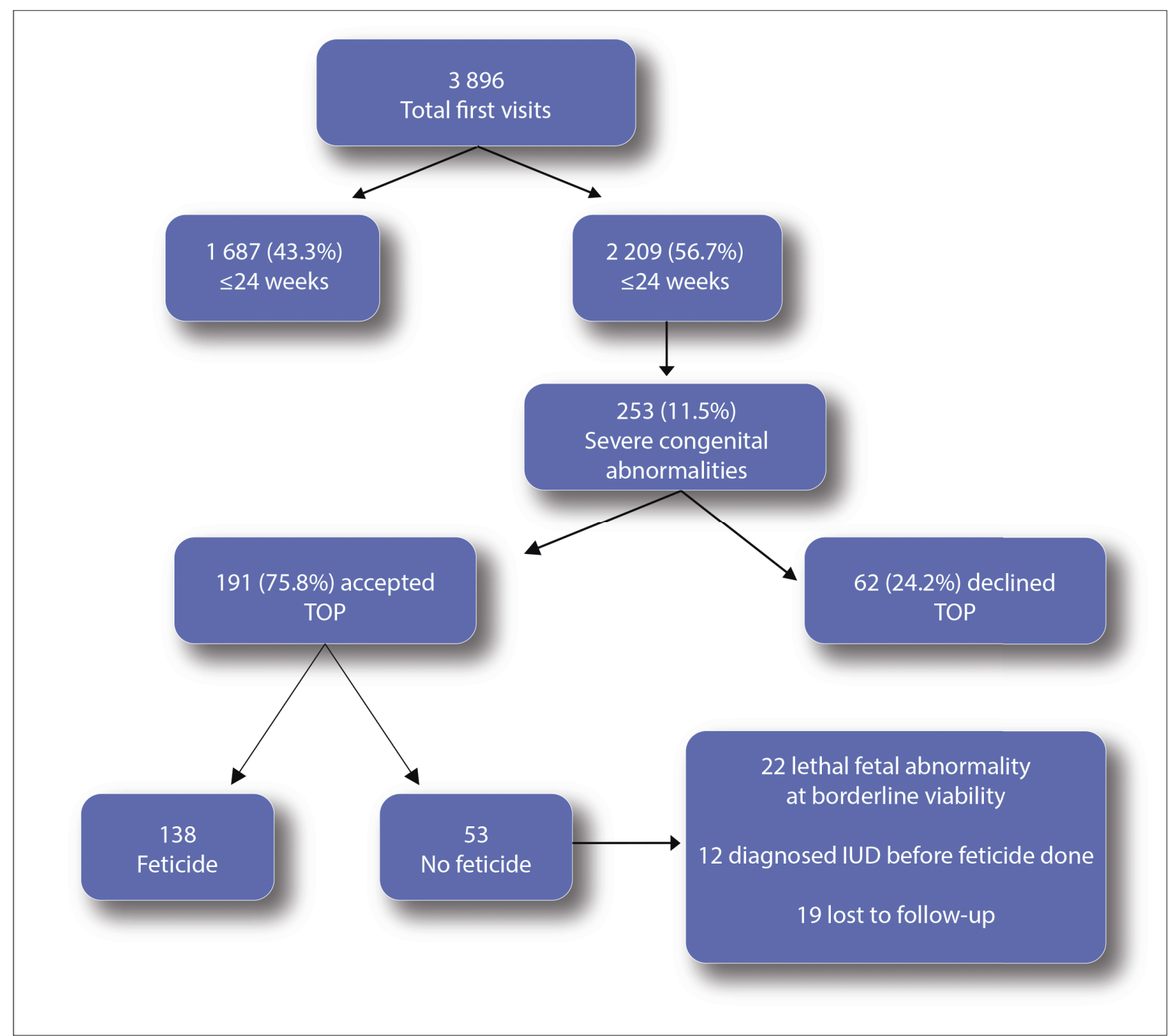

Fig. 1. Flow diagram of the women seen at the Fetal Unit during the 5-year study period.

\section{Results}

During the 5-year study period, 3896 women were referred to the Fetal Unit, of whom 2209 (56.7\%) were at $\geq 24$ weeks' gestation at first presentation. LTOP for severe congenital abnormalities was offered to 253 women (study group), of whom 191 (75.5\%) accepted (Fig. 1). The differences in the mean maternal age (26 years), parity (1) and GA at diagnosis (31+ weeks) between the accepted and declined groups were not significant.

The most frequent indications for LTOP were brain and spinal abnormalities (53.0\%), aneuploidy (20.6\%) and multiple congenital abnormalities (11.5\%). Table 1 compares the types and prevalences of fetal abnormalities and the rates of acceptance of LTOP between the groups. Although not statistically significant $(p=0.06)$, the differences between the groups that accepted or declined LTOP based on the type of fetal anomaly were clinically important.

Of the 134 brain anomalies, 52 were hydrocephalus; 38 of these women accepted LTOP, and half had concomitant cephalocentesis. There were 27 cases of spina bifida, and in this group 16 women accepted LTOP. Fig. 2 shows the distribution of the types of aneuploidy. Of women with a confirmed diagnosis of fetal aneuploidy,
67.3\% (35/52) accepted LTOP. The commonest aneuploidy was trisomy 18 (28/52), and of these women 20 accepted LTOP. There were 9 cases each of trisomy 21 and trisomy 13 .

\section{Outcomes of fetal congenital abnormalities}

Feticide by intracardiac KCl injection was performed in 138/191 cases in the accepted group (72.2\%) at a mean GA of 33 weeks (range 26 - 41 weeks). The mean interval between diagnosis and feticide was 10 days (range 0 - 42); 53 women accepted but did not have LTOP feticide. Of the 53, 22 had TOP between 24 and 26 weeks' gestation for a lethal anomaly detected at borderline viability, which all resulted in a stillbirth or death within 12 hours of birth. Twelve women booked for feticide presented with intra-uterine fetal death before the feticide - these cases included fetal anomalies complicated by fetal hydrops, severe growth restriction, and fetuses with multiple anomalies. The remaining 19 women who accepted LTOP did not return for the procedure, were lost to follow-up, or delivered before their procedure appointment.

The number of feticides performed per anomaly category was as follows: central nervous system 65 (excluding neural tube defects), neural tube defects 22 , aneuploidy 18 , multiple fetal abnormalities 17 , twin anomalies 5, skeletal 6, cardiac 3, and renal 2. Fetal asystole was 


\section{RESEARCH}

Table 1. Types and prevalence of all fetal abnormalities, with rates of acceptance of late termination of pregnancy

\begin{tabular}{llcc}
\hline & \multicolumn{2}{c}{ Decision on TOP, $\boldsymbol{n}(\%)$} & \multirow{2}{*}{ Total, $\boldsymbol{n}(\%)$} \\
\cline { 2 - 3 } Diagnosis & Accepted & Declined & $134(53.0)$ \\
\hline Brain and spine & $96(71.6)$ & $38(28.4)$ & $52(20.6)$ \\
Aneuploidy & $35(67.3)$ & $17(32.7)$ & $29(11.5)$ \\
MCA & $28(96.6)$ & $1(3.3)$ & $13(5.1)$ \\
Skeletal & $12(92.3)$ & $1(7.7)$ & $10(4.0)$ \\
Renal & $9(90.0)$ & $1(10.0)$ & $8(3.2)$ \\
Cardiac & $5(62.5)$ & $3(37.5)$ & $7(2.8)$ \\
Twins & $6(85.7)$ & $1(14.3)$ & 253 \\
Total, $N$ & $191(75.5)^{*}$ & $62(24.5)$ & \\
${ }^{*} p=0.06$. & & & \\
MCA $=$ multiple congenital abnormalities. & & &
\end{tabular}

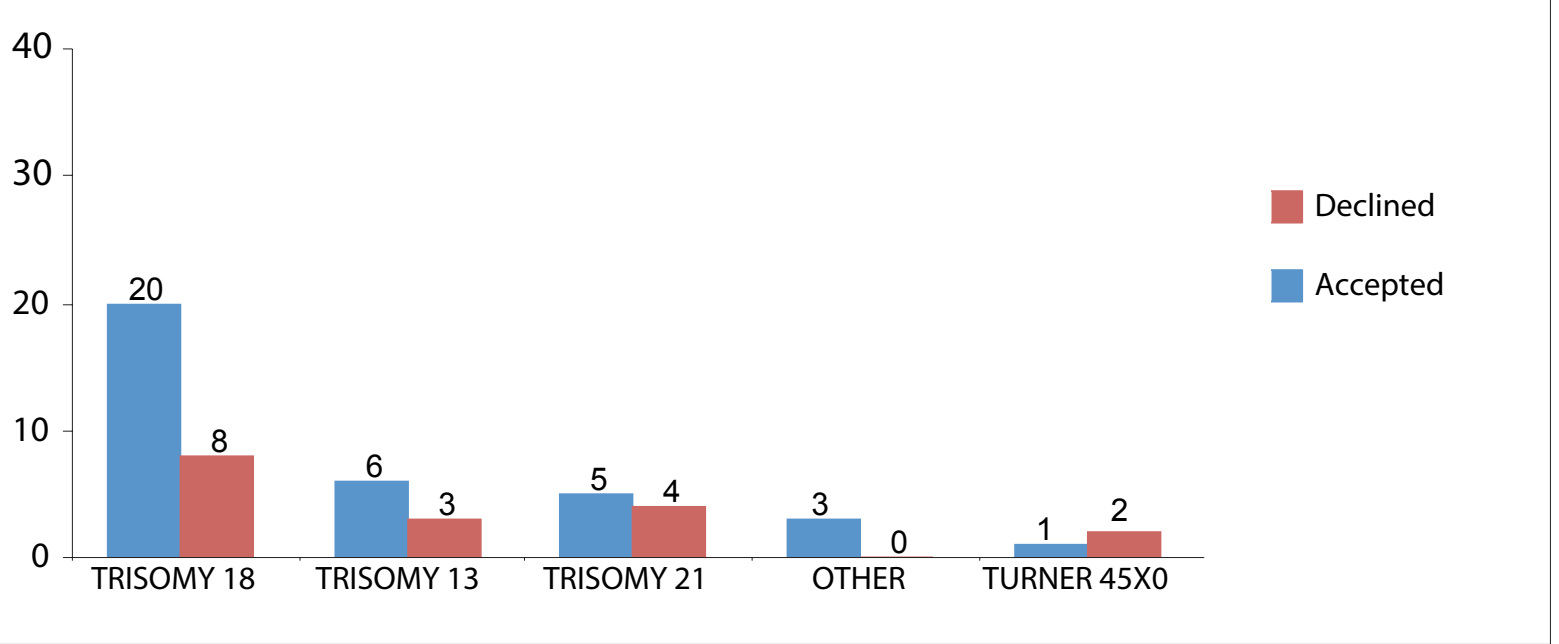

Fig. 2. Distribution of aneuploidy and rate of acceptance of late termination of pregnancy between the groups $(\mathrm{n}=52)$.

achieved in all cases within 2 minutes of intracardiac $\mathrm{KCl}$ injection with mean volumes of $8 \mathrm{ml}$ (GA 24 - 25 weeks), $10 \mathrm{ml}$ (26 - 30 weeks) and $13 \mathrm{ml}$ (>30 weeks) ( $p=0.001)$ (Table 2). The average duration of the procedure was 12 minutes (range 6 - 25 minutes). Only one-third of the women presented for the 6-week follow-up visit. Feticidedelivery intervals, modes of delivery, and perinatal outcomes for those who opted to continue with the pregnancy were therefore not evaluated. No maternal complications were reported. All women who had a feticide delivered stillbirths.

\section{Discussion}

In South Africa, there are no known national data on LTOP and feticide for fetal abnormalities, nor are there guidelines and legislature on feticides. In the literature, establishing clear guidelines is an issue of debate. ${ }^{12}$ Despite the provision of free maternity services in South Africa, many women book late for antenatal care. This situation is compounded by lack of resources and skills in performing obstetric scans, missed early diagnosis, late evolution of certain abnormalities, delay in referral to tertiary centres and late decision regarding TOP when a severe congenital abnormality is diagnosed. It is therefore not surprising that the number of fetal abnormalities detected after clinical viability is high compared with affluent countries. Further management of such pregnancies poses a major decision-making challenge for the pregnant woman and her family, and also for the physician and other health workers who must manage the pregnancy.

Several academic units in South Africa perform feticide for severe fetal abnormalities detected after 22 - 24 weeks' gestation. In KZN, the Fetal Medicine Unit at IALCH has established guidelines for LTOP. Contrary to the belief that religion plays a major role in end-of-life decision for the unborn baby, we found that the decisionmaking processes was not influenced by maternal age, parity, social status or GA at diagnosis of fetal anomaly. This retrospective audit could not analyse the influence of partners and other family members on the decision making.

Our highest prevalence of severe fetal abnormalities was in primigravidas (38.7\%) and those between ages of 15 and 24 (43.5\%) Older women are at higher risk for fetal abnormalities, and in one-fifth of our cases of severe fetal abnormalities the mother was over 35 years old. The lower prevalence of fetal abnormalities in our patients aged $>35$ years may be due to the smaller number of women in this age category and the nature of the anomalies. 
Table 2. Dosage of $\mathrm{KCl}(\mathrm{ml})$ according to gestational age category $(N=138)$

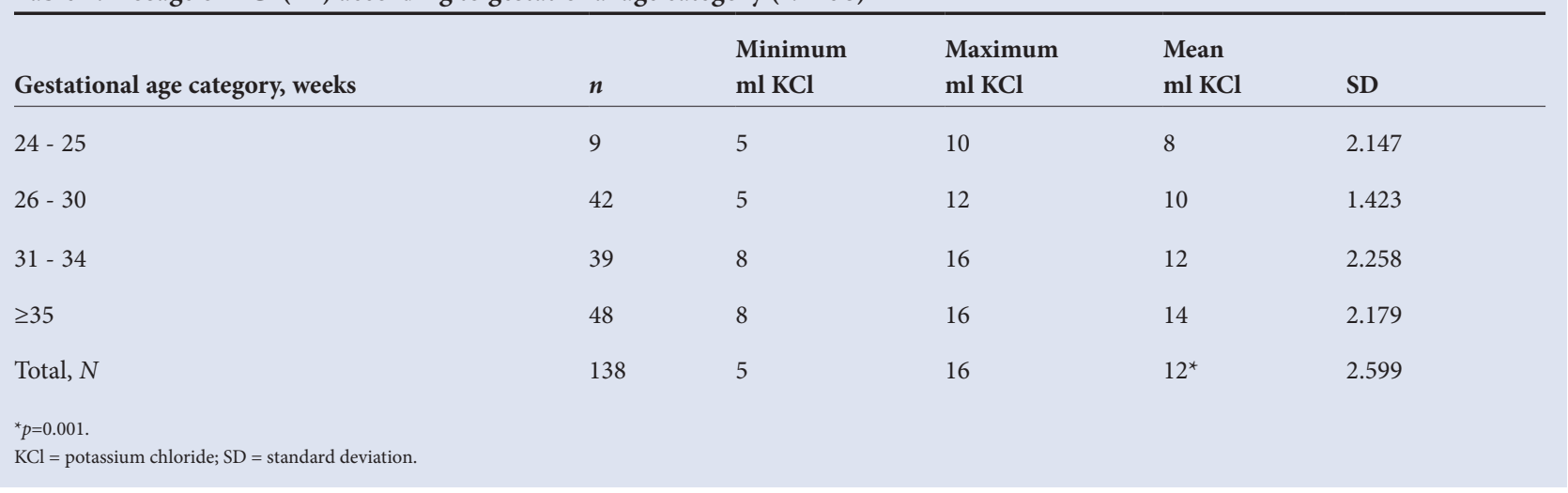

Structural abnormalities were more common than aneuploidy in late pregnancy. The commonest fetal abnormality involved the CNS, with hydrocephalus being the commonest brain abnormality. The highest acceptance rate for LTOP was in women between the ages of 25 and $34(80.6 \%)$ and with 2 or fewer children. Women aged $>35$ years and those with more than 3 children had the lowest acceptance rates $(66.0 \%$ and $55.9 \%$, respectively). Eighty-one per cent of women at GA $24-25$ weeks and $71.6 \%$ at GA $>35$ weeks accepted TOP $(p>0.05)$. Although these figures are not significantly different, it seems that, as human nature would predict, the more advanced the gestation, the more difficult the decision-making process becomes. The type of fetal abnormality also affected acceptance of LTOP by the woman and her family. Although not statistically significant, the acceptance rate was higher for the more lethal anomalies such as multiple congenital abnormalities (90.0\%) than for less lethal ones such as spina bifida (59.2\%) or anomalies where the prognosis is less certain such as complex cardiac anomalies $(62.5 \%)(p=0.086)$.

Concerning the procedure, our results show a direct correlation between the dose of $\mathrm{KCl}$ and advancing gestation at the time of performance of feticide. Various methods of feticide with differing success rates are described, including intra-umbilical lidocaine and $\mathrm{KCl},{ }^{8-13}$ intracardiac $\mathrm{KCl},{ }^{9}$ cardiac tamponade with normal saline ${ }^{10}$ and aspiration of blood from the fetal heart. ${ }^{11}$ One group ${ }^{5}$ also uses intra-umbilical sufantenil for fetal analgesia before administering the lethal drug. As fetal death with intracardiac $\mathrm{KCl}$ injection is rapid, the role of fetal analgesia is uncertain. We concur with the Queen Charlotte's group that intracardiac injection of $\mathrm{KCl}$ is an effective method of feticide in LTOP for severe congenital abnormalities. ${ }^{9}$

To our knowledge, no studies have reported prevalence of fetal abnormalities and the indications for and acceptance rate of LTOP in low- and middle-income countries. We hope to increase public awareness of the available treatment options when a severe or complex abnormality is detected at a late gestation and to provide appropriate counselling for couples and families. This study also highlights the need for our health system to enhance professional support for women who request LTOP.

\section{Limitations}

The safety and efficacy of the feticide procedure was based on the experience and expertise of one fetal medicine specialist (LG), who performed all the procedures. The number of women with fetal abnormalities in this study was also not a true reflection of the incidence of fetal abnormalities/birth defects in the province of KZN, since not every woman with a fetal anomaly was seen at this unit. The distant geographical locations in KZN resulted in some women being lost to follow-up. In addition, the perinatal outcomes of women who declined termination were not evaluated and autopsies for severe fetal anomalies were not performed, for reasons beyond the scope of this paper.

\section{Conclusion}

Our study shows that the type of fetal anomaly rather than the demographic details of the patient or the GA at diagnosis affect the decision to accept or decline LTOP. Our most frequent indication for LTOP was brain abnormalities, followed by chromosomal abnormalities. Intracardiac $\mathrm{KCl}$ administered under direct vision induces immediate asystole and appears to be a relatively safe and effective method for LTOP. However, $\mathrm{KCl}$ is associated with potentially serious maternal side-effects. Further studies are required to determine the minimum dosage of intracardiac $\mathrm{KCl}$ to achieve the desired effect.

\section{References}

1. Choice on Termination of Pregnancy Act, Act 92 of 1996. www.info.gov.za/gazette/acts/1996/a92-96.htt (accessed 15 March 2008)

2. Woodrow NL. Termination review committees: are they necessary? Med J Aust 2003;179(2):92-94

3. Chervenak F, McCullough LB. Responsibly counselling women about the clinical management of pregnancies complicated by severe fetal anomalies. J Med Ethics 2012;38:397-398. [http://dx.doi. org/10.1136/medethics-2012-100491]

4. Napolitano R, Thilaganathan B. Late termination of pregnancy and foetal reduction for foetal anomaly. Best Prac Res Clin Obstet Gynecol 2010;24:529-537. [http://dx.doi.org/10.1016/j.bpobgyn.2010.02.004]

5. Graham RH, Mason K, Rankin J, Robson, SC. The role of feticide in the context of late terminatio of pregnancy: a qualitative study of health professionals' and parents' views. Prenatal Diagnosis 2009;29(9):875-881. [http://dx.doi.org/10.1002/pd.2297]

6. Deana G, Colarossia L, Lundeb B, Jacobsc AR, Porscha LM, Paula ME. Safety of digoxin for fetal demise before second-trimester abortion by dilation and evacuation. Contraception 2012;85:144-149. [http:// dx.doi.org/10.1016/j.contraception.2011.05.016

7. Dommergues M, Benachi A, Benifla JL, des Noettes R, Dumez Y. The reasons for termination of pregnancy in the third trimester. Br J Obstet Gynaecol 1999;106(4):297-303. [http://dx.do. org/10.1111/j.1471-0528.1999.tb08265.x]

8. Senat MV, Fischer C, Bernard JP, Ville Y. The use of lidocaine for fetocide in late termination of pregnancy. BJOG 2003;110(3):296-300. [http://dx.doi.org/10.1046/j.1471-0528.2003.02217.x]

9. Pasquini L, Pontello V, Kumar S. Intracardiac injection of potassium chloride as method for feticide experience from a single UK tertiary centre. BJOG 2008;115(4):528-531. [http://dx.doi.org/10.1111/ .1471-0528.2007.01639.x]

10. Chen $\mathrm{CH}$, Hsieh HC, Tsai HD, Chen TH, Chen M. Cardiac tamponade: an alternative procedure for late feticide. Taiwan J Obstet Gynecol 2009;48(2):159-162. 10.1016/\$1028-4559(09)60278-3]

1. Beksac MS, Balci S, Ozlu T, Ozyuncu O. Selective feticide in dichorionic pregnancies with intracardiac blood aspiration: report of nine cases. J Perinat Med 2009;37(1):85-86. [http://dx.doi.org/10.1515/ JPM.2009.010]

12. Senat MV, Fischer C, Ville Y. Funipuncture for fetocide in late termination of pregnancy. Prenat Diagn 2002;22(5):354-356. [http://dx.doi.org/10.1002/pd.290]

3. Statham H, Solomou W, Green JM. Late termination of pregnancy: law, policy and decision making in four English fetal medicine units. Int J Obstet Gynecol 2006;113:1402-1411. [http://dx.doi.org/10.1111/j.14710528.2006.01144.x]

Accepted 10 September 2012 